\title{
Ecología y reproducción de Opsanus beta (Actinopterygii: Batrachoididae) en la Laguna de Alvarado, Veracruz, México
}

\author{
Jonathan Franco López¹, Alma G. Santes González¹, Luis G. Abarca Arenas², Carlos Bedia \\ Sánchez ${ }^{1}$, Héctor Barrera Escorcia ${ }^{1 \dagger}$, José A. Martínez Pérez ${ }^{3}$, Edgar Peláez Rodríguez ${ }^{1} \&$ \\ José L. Viveros Legorreta ${ }^{1}$ \\ 1. Laboratorio de Ecología, Facultad de Estudios Superiores, UNAM. Av. de los Barrios No. 1 Los Reyes Iztacala, \\ Tlalnepantla, Estado de México C.P.54090; jonfrancol@yahoo.com,biol_puma06@hotmail.com, cbedias@unam.mx, \\ chacagna@hotmail.com, huifire_pepe@yahoo.com.mx \\ 2. Instituto de Investigaciones Biológicas, Universidad Veracruzana. Av. Luis Castelazo Ayala s/n Col. Industrial Ánimas \\ C.P.91190, Xalapa, Veracruz, México; luisgaa@gmail.com \\ 3. Laboratorio de Zoología, Facultad de Estudios Superiores, UNAM. Av. de los Barrios No. 1 Los Reyes Iztacala, \\ Tlalnepantla, Estado de México C.P. 54090; jamp55@prodigy.net.mx
}

Recibido 04-III-2017. Corregido 10-VII-2017. Aceptado 09-VIII-2017.

\begin{abstract}
Ecology and reproduction of Opsanus beta (Actinopterygii: Batrachoididae) in the Alvarado Lagoon, Veracruz, Mexico. Opsanus beta is a permanent species in the Alvarado Lagoon System (ALS), Veracruz, and eventhough it is not of economic importance, it might be actively involved in the energy transfer of the system. Currently, there is no information about the reproductive and ecological characteristics of this common fish, so this work aimed to evaluate those aspects, and to contribute with some ecological features. For this, a total of six sampling sites were selected and studied from April 2008 to December 2012. Per site, we determined fish seasonality, feeding, sex ratio, gonadosomatic index, hepatosomatic and condition factors for both sexes, as well as the fecundity and length-weight relationships. We also considered some environmental parameters such as temperature, water temperature, depth, salinity and $\mathrm{pH}$. Our results on the environmental parameters analysis did not show significant differences for the study period; besides, salinity data confirmed an oligohaline environment, and the rest of the physico-chemical parameters, varied in accordance with the climatic pattern in the area. We captured a total of 519 fishes, 311 (59.9\%) males and 208 (41.1\%) females; the proportion of males resulted favorable against females throughout the study. The standard length found along the study period showed that the average was higher in males than in females. The trophic spectrum was composed of 13 food items, principally crustaceans, molluscs and fishes; in addition, cannibalism was observed over young individuals during March and April. The most advanced stages of gonadal maturation were recorded from November to March for males, and from November to February in females; generally, the Gonadosomatic index (IGS) recorded the highest values from November to April. The Hepatosomatic Index (IHS) and the Condition Factor (FC) presented an inverse relationship with the Gonadosomatic index (IGS). The fecundity of this species ranged from 96 to 428 oocytes per female. The weight-length relationship by sexes and seasons, showed a higher growth rate for males than for females, and an allometric growth type. The biological and ecological information of O. beta in ALS evidenced the importance of this species and its ecological role in the structure and dynamics of these aquatic communities. This species may be used as a model for future proposals dealing with the management and sustainable use of this ecosystem. Rev. Biol. Trop. 65 (4): 1381-1396. Epub 2017 December 01.
\end{abstract}

Key words: Opsanus beta, reproductive index, food, growth.

El Sistema Lagunar de Alvarado (SLA), se caracteriza por la interacción de diversos humedales que confluyen en él, estos son ecosistemas complejos, dinámicos y altamente productivos, pues no solo proveen de alimento, sino brindan refugio, y funcionan como áreas de reproducción y de crianza a un alto número de especies de invertebrados, peces, 
anfibios, reptiles y aves (Ramos, 1997), que participan activamente en la estructura trófica y energética del sistema lagunar. Sin embargo, aún cuando se ha reconocido la importancia de estos subsistemas para el mantenimiento de la multiplicidad de funciones ecológicas que se les ha conferido (Wood, Odum, \& Zieman, 1969; Costanza et al., 1997), y de las complejas interacciones entre los organismos y el ambiente (Yáñez-Arancibia, 1978), los estudios a nivel de especie con una función ecológica importante son escasos y se requiere documentar su participación en la dinámica propia de este ecosistema.

De las diversas especies de peces que habitan este sistema, los representantes de la familia Batrachoididae utilizan tanto las zonas de vegetación sumergida, como áreas de manglar, zonas de ostión y áreas fangosas (Collette \& Russo, 1981; Pereira, Silva, Costa, \& Costa, 2011) para completar su ciclo de vida. En el SLA, se encuentra Opsanus beta, que es una especie eurihalina, carnívora y permanente que ocupa diversos componentes del hábitat presentes en estas áreas como son envases, troncos caídos u oquedades en el sedimento para completar su ciclo reproductivo, característica que comparte con otras especies espeleofilas reconocidas (Balon, 1975; Roux, 1986; Chandler, Sanders, \& Landry, 1985; Tupper \& Boutilier, 1995). La especie originalmente fue descrita como Batrachus tau beta, por los ictiólogos Goode y Bean (1880), también se reconoció por el sinónimo de Opsanus vandeuseni, Fowler, 1939, aunque ahora se conoce como Opsanus beta, Goode y Bean (1880), nombre científicamente válido y usado actualmente. En el lenguaje común éste pez es conocido como pez sapo (toadfish), pez sapo del golfo (gulf toadfish), pez perro (dogfish), pez del fango (mudfish), perro de ostras (Oysterdog), ó sapo de boca blanca (Collette, Aiken, \& Polanco-Fernández, 2015). De los pocos estudios realizados con esta especie destacan un registro como especie exótica en las costas de Brasil (Caires, Pichler, Spach, \& Ignacio, 2007), y una evaluación del ritmo de crecimiento realizada en Florida, E.U. (Malca, Barimo, Serafy, \& Walsh, 2009).
Esta especie es un habitante permanente en los sistemas estuarinos del estado de Veracruz, y aunque no presenta importancia comercial, se considera que su papel ecológico puede ser fundamental en la transformación y transporte de energía al interior del ecosistema. El presente trabajo se desarrolló con la finalidad de evaluar los principales aspectos de la biología de esta especie en el SLA, que incluyen estacionalidad, alimentación, proporción de sexos, índices gonadosomático, hepatosomático, factor de condición, fecundidad y la relación peso-longitud para ambos sexos, con base en los registros obtenidos de esta especie para el período de abril 2008 a diciembre 2012.

\section{MATERIALES Y MÉTODOS}

Los ejemplares de Opsanus beta se recolectaron en seis sitios del Sistema Lagunar de Alvarado (que comprendieron las lagunas de Camaronera, Buen País y Alvarado), con el uso de un chinchorro playero, de $50 \mathrm{~m}$ de largo, $2.5 \mathrm{~m}$ de altura y luz de malla de una pulgada, en las distintas temporadas climáticas de Secas (marzo-abril), lluvias (junio-septiembre) y Nortes (noviembre-febrero) en el período de abril de 2008 a diciembre de 2012 (Fig. 1). Los organismos fueron fijados in situ con formol neutro al $10 \%$, se inyectaron en la zona abdominal, con la finalidad de frenar los procesos digestivos y para facilitar la acción del fijador en la gónada, y la resistencia a la manipulación posterior (Arceo, Franco, Gretchen, \& Chávez, 2004).

Parámetros fisicoquímicos: De forma complementaria, se registraron diversos parámetros ambientales, profundidad (profundímetro portátil digital PS-7), temperatura ambiental (termómetro Taylor de -10 a $50{ }^{\circ} \mathrm{C}$ ), temperatura del agua, salinidad y $\mathrm{pH}$ (medidor multiparámetro YSI-63), y oxígeno disuelto (oxímetro YSI-55) en cada sitio de colecta. Para determinar si se presentaban diferencias significativas en el comportamiento físico-químico entre las estaciones de colecta, por mes y entre años de estudio, los datos de cada parámetro: 


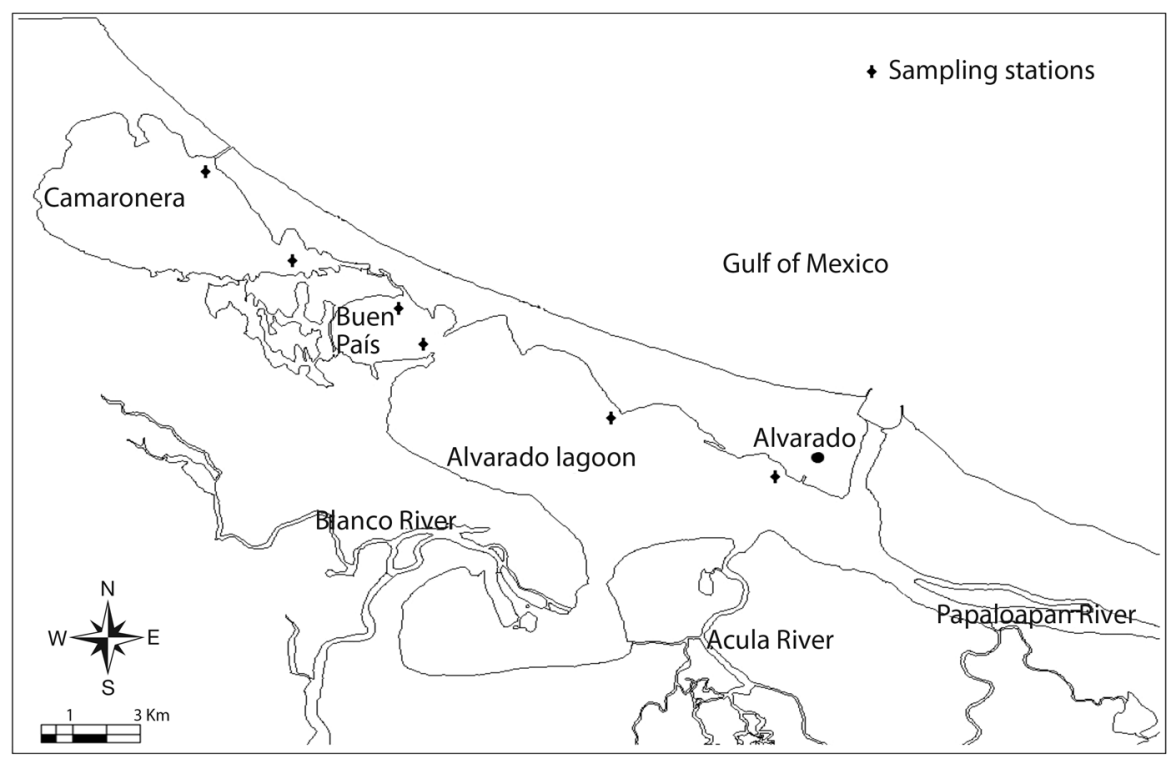

Fig. 1. Sitios de recolecta para Opsanus beta en el Sistema Laguna de Alvarado.

Fig. 1. Collection sites for Opsanus beta in the Alvarado Lagoon System.

temperatura ambiente, temperatura del agua, profundidad, transparencia, salinidad y oxígeno, se sometieron a un análisis ANOVA de dos vías.

Parámetros biológicos: En el laboratorio, se registraron los datos morfométricos de cada organismo, tales como longitud estándar (cm) y peso total $(\mathrm{g})$, y se compararon para el período de estudio con una prueba de t-student. También se obtuvo el peso del hepatopáncreas y de la gónada, así como el peso eviscerado (Rodríguez, 1992). Para el análisis del contenido alimenticio se extrajo el tracto digestivo, vaciándolo en una caja Petri, y se reconocieron los items alimenticios por tipo y número, por el método porcentual (Bagenal, 1978). Los resultados obtenidos se procesaron por el Índice de importancia relativa, ajustando su estimación a $100 \%$.

La proporción de sexos se calculó dividiendo el número total de machos entre el número total de hembras por mes y para todo el periodo de estudio. Para determinar si la proporción de sexos se ajustaba a la distribución teórica de 1:1, se utilizó la prueba de chi cuadrada $\left(X^{2}\right)$ de acuerdo con Zar (2010).

Se determinó el grado de maduración sexual para ambos sexos con la escala de Nikolsky (1963), y para el caso de las hembras con estadios de maduración avanzada, se registró el conteo de ovocitos por gónada. Se complementó esta información de la especie con la estimación de los índices Gonadosomático y Hepatosomático, Maddock y Burton (1999) así como el factor de condición de acuerdo a las siguientes relaciones:

\footnotetext{
Índice gonadosomático $(\mathrm{IGS})=$

Peso Gonádico (g) / Peso Total (g)* (100)

Índice hepatosomático (IHS) =

Peso Hepatopáncreas (g) / Peso Total (g) * (100)

Factor de condición $(\mathrm{FC})=$

Peso Total (g) / Longitud Patrón (cm)* (100)

La relación peso-longitud se calculó con base a los registros de talla y peso por temporada climática, se utilizó la ecuación de Le Cren (1951), que permite ajustar los valores
} 
a través de una relación potencial del peso respecto de la longitud de los individuos, a partir de la ecuación:

$$
\mathrm{W}=\mathrm{a} \mathrm{L}^{\mathrm{b}}
$$

donde:

$\mathrm{W}=$ peso $(\mathrm{g})$

$\mathrm{L}=$ longitud $(\mathrm{cm})$

$\mathrm{a}=$ ordenada al origen

$\mathrm{b}=$ pendiente (factor de alometría)

La obtención de las constantes de esta ecuación, se realizó a partir de su linearización logarítmica, quedando como:

$$
\log W=\log a+b \log L
$$

Los valores de b de ambos sexos se compararon respecto del valor teórico de 3 , con una prueba de $\mathrm{t}$ de student $(\alpha<0.05)$, para determinar el tipo de crecimiento (isométrico o alométrico) de la especie. Todos los análisis estadísticos se realizaron con ayuda de los programas STATGRAPHICS XV.1 y XLSTAT 3.02 (Addinsoft, 2009).

\section{RESULTADOS}

Parámetros físico-químicos: El registro de los parámetros físicoquímicos obtenidos de las seis estaciones presentó pequeñas variaciones en cada uno de los meses de colecta y que están directamente relacionados con las temporadas climáticas (Cuadro 1), donde las variaciones de la temperatura del agua, la salinidad y la concentración de oxígeno disuelto entre los años de muestreo no fueron significativas (ANOVA, $\mathrm{F}_{5,24}=2.621, \mathrm{p}<0.998$ ).

El comportamiento de la temperatura del agua presentó mayores valores en los meses que corresponden a las temporadas de secas y lluvias con valores superiores a los $26{ }^{\circ} \mathrm{C}$, en tanto que las temperaturas bajas se presentaron en los meses de la temporada de nortes (Cuadro 1).

La mayor salinidad (8.6 g / L), se presentó en los meses de la temporada de secas, mientras 
que en las temporadas de lluvias y nortes, se registraron las menores salinidades (Cuadro 1). Por su parte, para el oxígeno disuelto la mayor concentración (8.5 ppm), se presentó en los meses de nortes, registrando las menores concentraciones en los meses que comprendieron las temporadas de secas y lluvias (Cuadro 1).

Parámetros biológicos: Se capturó en total 519 organismos a lo largo del período de estudio, de los cuales 311 (59.9\%) fueron machos y 208 (41.1\%) hembras (Fig. 2). Se capturó el mayor número de ejemplares en los meses de abril, noviembre, diciembre y marzo (Fig. 2). Las tallas y pesos registrados por sexo fueron, para el caso de los machos de los 4.1 a $23.8 \mathrm{~cm}$., con un peso de 0.7 a $426.8 \mathrm{~g}$; en tanto que para las hembras, las tallas oscilaron de 3.5 a $23 \mathrm{~cm}$., de longitud patrón y un peso de 2.6 a 320.7 g. En los distintos meses, los machos presentaron una longitud y peso promedio mayor que los valores determinados para hembras, $\mathrm{t}$ $(517)=-7.1203,-5.5746 \mathrm{p}<0.0001$ (Fig. 3A y Fig. 3B).

Se pudo determinar que el espectro trófico de $O$. beta en el SLA se compone de trece tipos alimenticios, destacando los crustáceos, moluscos y peces. Particularmente, en esta última categoría, se observó la práctica de canibalismo sobre los individuos jóvenes en los meses que comprende la temporada de secas.
Para los meses de lluvias y nortes, el número de tipos alimenticios se incrementó, al incluir otras especies de peces como Citharichthys spilopterus, Gobionellus oceanicus y otros no identificados, así como el bivalvo Mytilopsis sp. (Cuadro 2).

La proporción de sexos en general favoreció a los machos, y presentó diferencias significativas de la relación 1:1 esperada, con valores de 1.49:1 por cada hembra en el periodo de estudio $X^{2}(1, \mathrm{~N}=519)=3.92 \mathrm{p}<0.05$; de forma mensual, los resultados oscilaron de 1.29:1 M:H, en febrero a 2.2:1 en abril y de 2.09:1 en diciembre $X^{2}(1, \mathrm{~N}=37$ a 90$)=3.92$ a $14.4 \mathrm{p}<0.05$. Los únicos meses en que la proporción de sexos favoreció a las hembras fueron noviembre con 1:1.14 M:H y enero con $\left.1: 1.03 X^{2}(1, \mathrm{~N}=118,57)=0.36,0.04 \mathrm{p}<0.05\right)$

Los estadios de maduración registrados a lo largo del estudio por mes para $O$. beta, permitieron reconocer que, en el caso de los machos, las etapas de maduración más avanzadas (IV y V) se presentaron desde noviembre a marzo (Fig. 4A). Por su parte, para el caso de las hembras, las etapas más avanzadas de maduración se presentaron en el período de noviembre a febrero (Fig. 4B), y se observó gónadas desovadas en enero y febrero.

Los resultados del índice gonadosomático por sexo, se sometieron a un análisis de varianza; no se encontró diferencias significativas

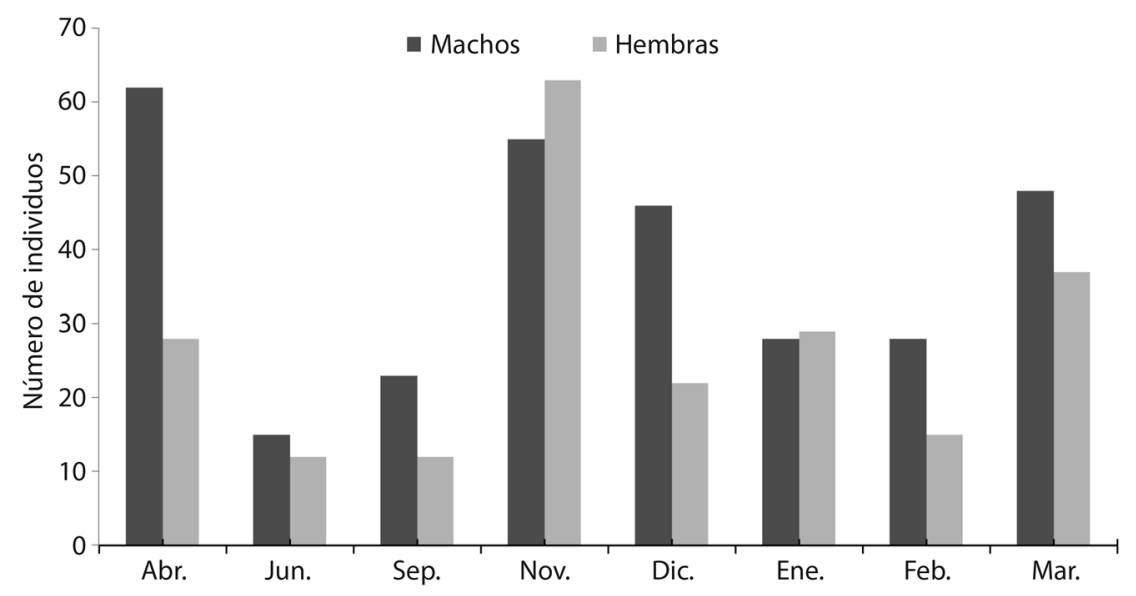

Fig. 2. Abundancia de machos y hembras de Opsanus beta por mes para el período 2008-2012. Fig. 2. Abundance of males and females of Opsanus beta per month for the period 2008-2012. 
CUADRO 2

Comportamiento alimenticio de Opsanus beta en porcentaje del IIR para el período 2008-2012

TABLE 2

Opsanus beta food composition as a percentage of IIR for the 2008-2012 period

\begin{tabular}{lcccccccc}
\multicolumn{1}{c}{ Tipo alimenticio/mes } & ABR & JUN & SEP & NOV & DIC & ENE & FEB & MAR \\
Callinectes sp. & 50.2 & 48.4 & 49.3 & 61.5 & 78.4 & 61.2 & 62.5 & 69.3 \\
Neritina virginea & 12.3 & 13.1 & 5.3 & 3.4 & 1.5 & 8.3 & 0 & 0 \\
Ruppia maritima & 9.6 & 21.4 & 6.2 & 4.1 & 4.6 & 3.6 & 0 & 8.5 \\
Detritus & 10.4 & 4.5 & 6.8 & 22.3 & 3.6 & 8.5 & 7.1 & 8.4 \\
Opsanus beta & 13.1 & 0 & 0 & 0 & 0 & 0 & 0 & 10.7 \\
Cardisoma sp. & 0 & 4.1 & 25.4 & 0 & 0 & 5.2 & 22.8 & 0 \\
Rangia sp. & 2.1 & 3.2 & 0 & 0 & 0 & 0 & 4.4 & 0 \\
Macrobrachium sp. & 0 & 3.3 & 3.8 & 0 & 2.5 & 5.1 & 0 & 0 \\
Citharichthys spilopterus & 0 & 0 & 0 & 0 & 3.4 & 0 & 0 & 0 \\
Anfípodos & 0 & 0 & 0 & 0 & 0 & 4.5 & 0 & 0 \\
Peces n.i. & 0 & 0 & 0 & 5.4 & 0 & 3.6 & 3.2 & 0 \\
Gobionellus oceanicus & 0 & 0 & 0 & 3.3 & 3.6 & 0 & 0 & 0 \\
Mytilopsis sp. & 2.3 & 2 & 3.2 & 0 & 2.4 & 0 & 0 & 3.1 \\
\hline
\end{tabular}
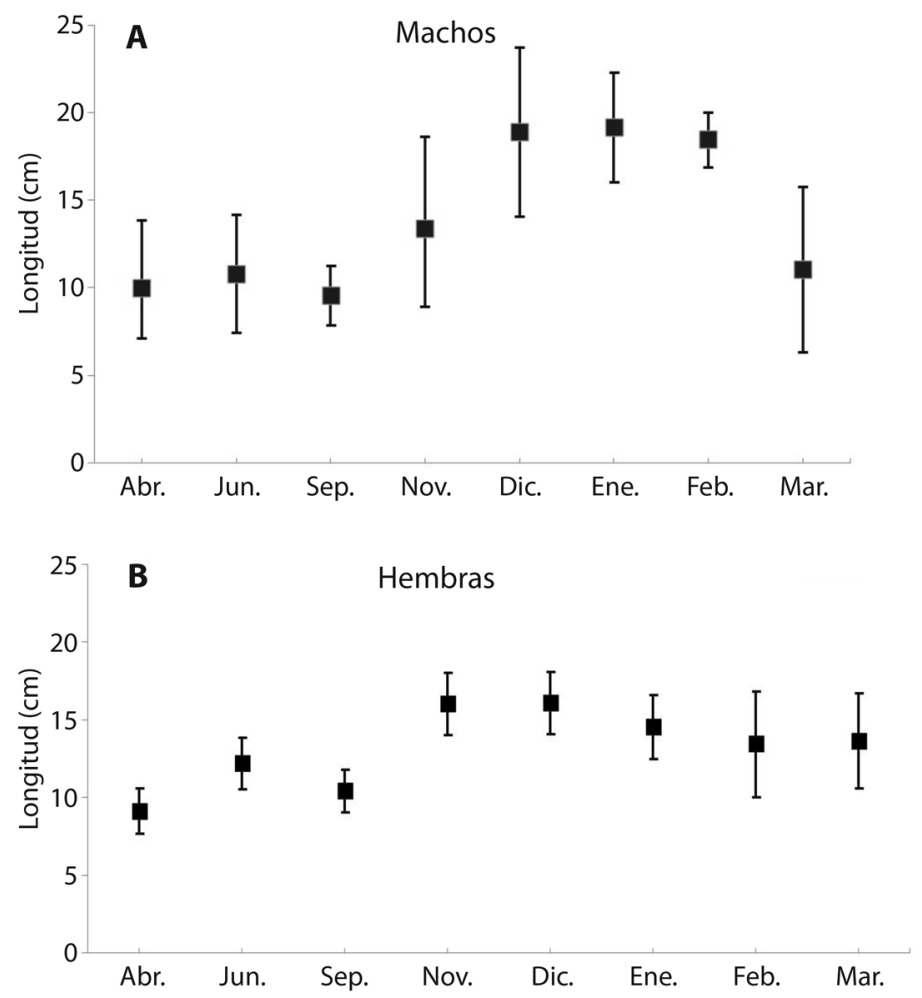

Fig. 3. Comportamiento de la longitud patrón promedio (+ / - d.e.) para machos (A) y hembras (B) en los meses en los que se capturó Opsanus beta.

Fig. 3. Average pattern length behavior (+/ - s.d.) for males (A) and females (B) in the months in which Opsanus beta was captured. 

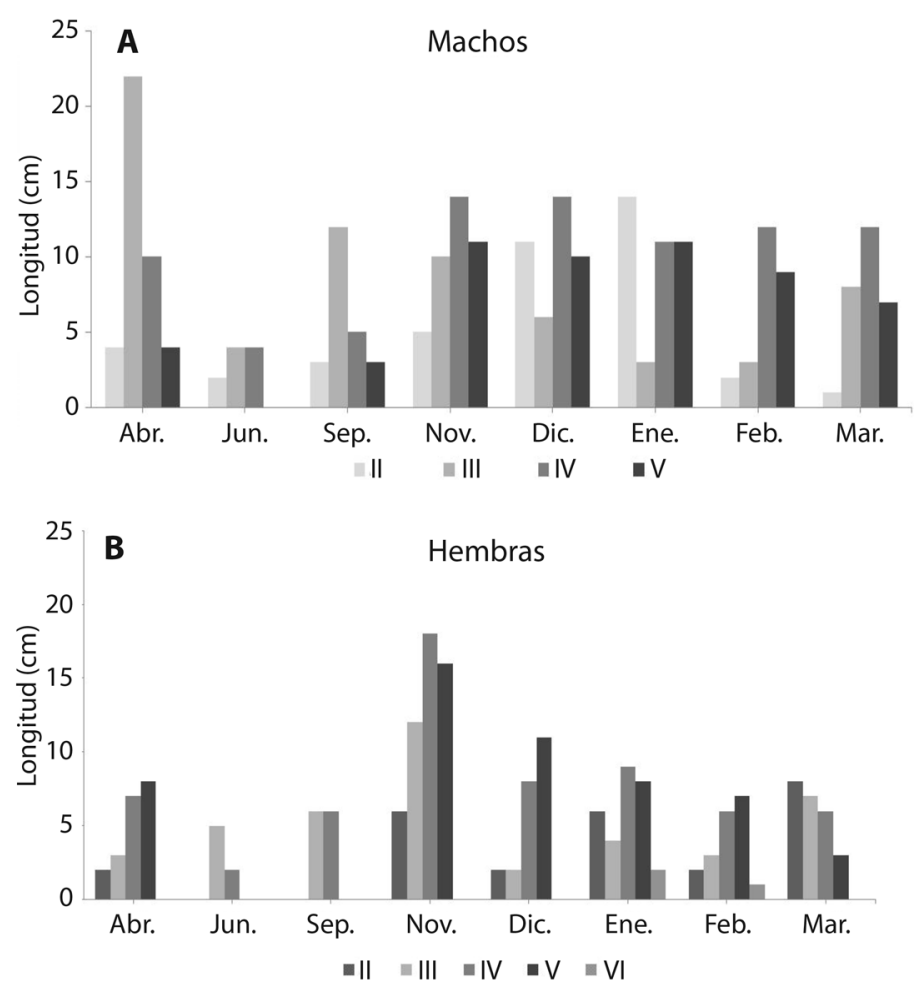

Fig. 4. Comportamiento de las etapas de maduración para machos (A) y hembras (B) de Opsanus beta por mes en el SLA en el período de estudio.

Fig. 4. Behavior of maturation stages for males (A) and females (B) of Opsanus beta per month in SLA in the study period.

entre los meses y años del estudio (ANOVA, $\left.\mathrm{F}_{7,32}=2.313, \mathrm{p}<0.102\right)$. Este índice presentó el comportamiento siguiente (Fig. 5A y Fig. 5B), para el caso de los machos, los valores más altos se registraron en los meses de noviembre a febrero, y para las hembras de noviembre a marzo.

Para el caso del índice hepatosomático, se aplicó el mismo tratamiento para ambos sexos, y no se registró diferencias significativas entre los meses y años considerados (ANOVA, $\mathrm{F}_{7,32}$ $=2.313, \mathrm{p}<0.160)$. El comportamiento promedio para cada sexo se muestra en las (Fig. 6); los valores más altos para los machos se presentaron en los meses de junio a noviembre, y para las hembras de junio a diciembre.

Por su parte, el factor de condición para $O$. beta registrado para cada sexo fue de 2.1 a 3.5 para los machos, con los valores más altos en los meses de junio a noviembre, y de 2.0 a 3.06 para las hembras, con los mayores valores en los meses de junio a diciembre (Fig. 7).

Para el caso de las hembras, los estadios de maduración avanzados donde se realizó el conteo de ovocitos por gónada, comprendió las etapas IV y V de madurez gonádica, este conteo se realizó en 106 hembras, registrando el número de ovocitos en la gónada izquierda y en la derecha. Los resultados arrojaron que el número de ovocitos por hembra, varía de 96 a 428, Cuadro 3, los resultados de ambas gónadas para las dos etapas se compararon con una prueba de t-student, obteniendo que en ambas etapas no se presentaron diferencias significativas $\left(\mathrm{t}_{\mathrm{IV}}(70)=1.938 \mathrm{p}<0.055 ; \mathrm{t}_{\mathrm{V}}(100)=\right.$ $0.338 \mathrm{p}<0.736$ ).

La estimación de la relación peso-longitud se calculó por temporada para cada sexo, las constantes obtenidas indicaron un crecimiento de tipo alométrico negativo, Cuadro 4. 

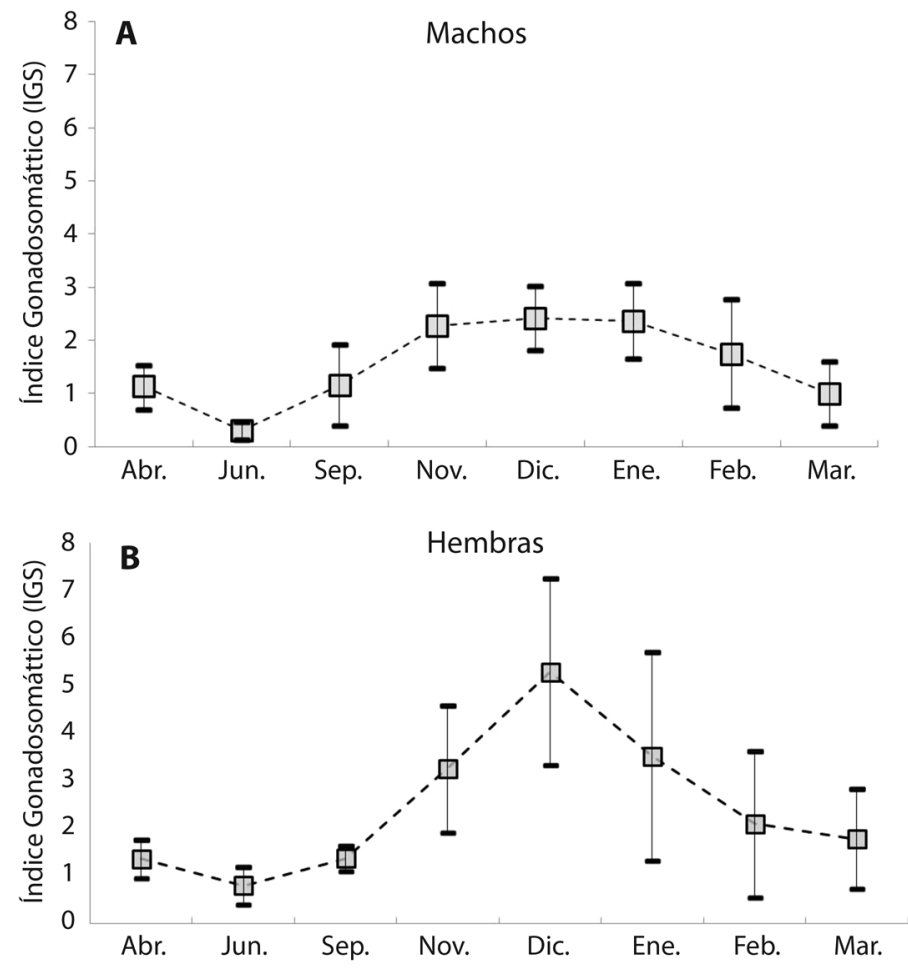

Fig. 5. Comportamiento del Índice Gonadosomático (+ / - d.e.) para machos (A) y hembras (B) de Opsanus beta en el SLA por mes en el período de estudio.

Fig. 5. Behavior of the Gonadosomatic Index (+/ - s.d.) for Opsanus beta males (A) and females (B) in the SLA for month in the study period.

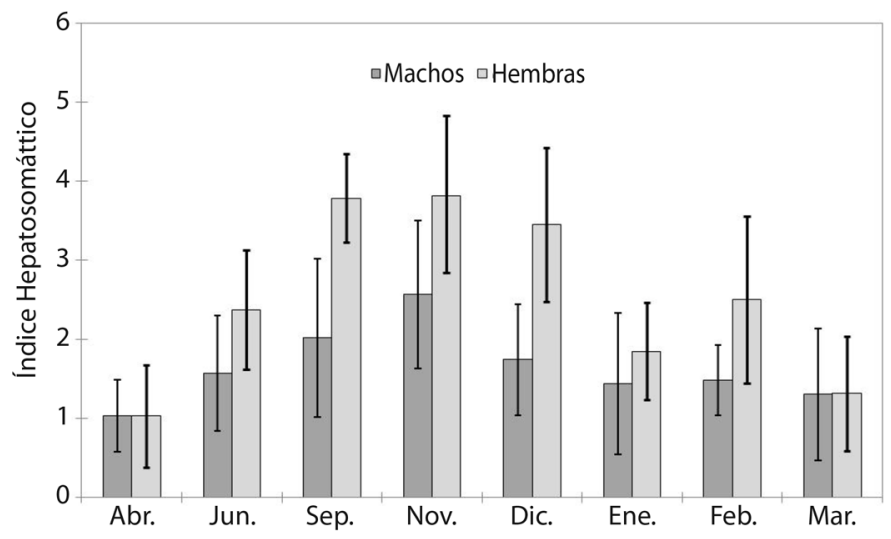

Fig. 6. Comportamiento del Índice Hepatosomático (+/ - d.s.) para machos y hembras de Opsanus beta en el SLA por mes. Fig. 6. Behavior of the Hepatosomatic Index (+/ - s.d.) for Opsanus beta males and females in the SLA for month. 


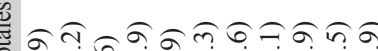

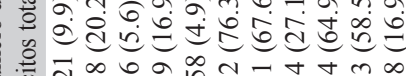

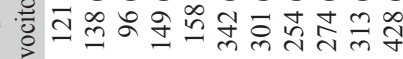

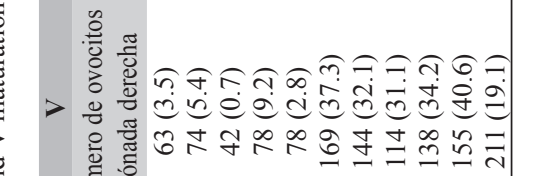

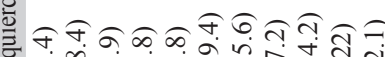

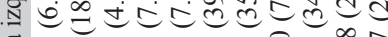

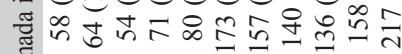

○ิ

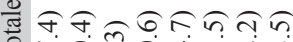

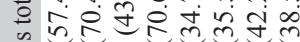

要

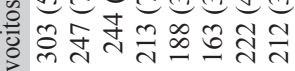

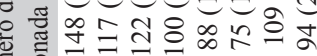

Por su parte, el ajuste realizado de forma total, correspondió a 311 machos y 208 hembras (Fig. $8 \mathrm{~A}$ y Fig. 8B), lo que en ambos casos, también resultó en un crecimiento de tipo alométrico negativo con base en la prueba de t-student.

\section{DISCUSIÓN}

Se ha reconocido que la variabilidad de los parámetros hidrológicos, en particular de este tipo de sistemas, es controlada por el viento, las mareas y la profundidad (Smith, 1997). Al respecto, Morán et al. (2005), mencionan que el comportamiento de los parámetros físicoquímicos en el sistema lagunar de Alvarado, puede explicarse por las variaciones climáticas y meteorológicas en la región, que permiten reconocer las temporadas de secas con un aumento de temperatura y aumento de la salinidad, lluvias con un incremento de escurrimientos continentales y una disminución de la salinidad, y los nortes donde predominan los vientos fríos y un ligero incremento de la salinidad provocado por la influencia marina hacia este sistema. Este comportamiento ocasiona que las condiciones hidrológicas del sistema correspondan a un ambiente oligohalino en la mayor parte del año, cambiando a condiciones mesohalinas en la época de secas. El análisis temporal de los parámetros ambientales no mostró diferencias significativas entre los meses durante el período de estudio, de esta forma, el comportamiento de la salinidad registrada a lo largo del estudio, correspondió a un ambiente oligohalino con registros de salinidad de $4.5 \mathrm{~g} / \mathrm{L}$., en junio a $9.7 \mathrm{~g} / \mathrm{L}$ en marzo, el resto de los parámetros fisicoquímicos registrados en el presente trabajo presentaron variaciones que pueden explicarse por el patrón climático en el área, donde, como es el caso de la temperatura y su variación de acuerdo a la época del año. Por su parte la concentración de oxígeno disuelto en las diferentes estaciones de muestreo y en el período de estudio, registró variaciones no significativas y que están directamente relacionadas con las temporadas climáticas como ha sido reportado previamente (Contreras \& Warner, 2004; Morán et al., 2005; 
CUADRO 4

Constantes de la relación Peso-Longitud por temporada y sexo para Opsanus beta, (n) número de individuos,

(b) pendiente, (a) ordenada al origen, $\left(\mathrm{R}^{2}\right)$ Coeficiente de determinación (Es) Error estándar

TABLE 4

Constants of the relationship Weight-Length by season and sex for Opsanus beta, (n) number of individuals, (b) slope,

(a) intercept, $\left(\mathrm{R}^{2}\right)$ Coefficient of determination, (Es) Standard error

\begin{tabular}{lccccc}
\multicolumn{1}{c}{ MACHOS } & $\mathrm{n}$ & $\mathrm{b}$ & $\mathrm{a}$ & $\mathrm{R}^{2}$ & Es \\
SECAS & 109 & 2.86 & 0.027857 & 0.8426 & 0.2108 \\
LLUVIAS & 37 & 2.57 & 0.08652 & 0.8145 & 0.1752 \\
NORTES & 165 & 2.74 & 0.05331 & 0.9459 & 0.1059 \\
TOTAL & 311 & 2.82 & 0.03896 & 0.8717 & 0.1794 \\
$\quad$ HEMBRAS & & & & & 0.1324 \\
SECAS & 62 & 2.65 & 0.062 & 0.9234 & 0.1195 \\
LLUVIAS & 24 & 2.66 & 0.07384 & 0.9048 & 0.0899 \\
NORTES & 122 & 2.85 & 0.0415 & 0.9239 & 0.1106 \\
TOTAL & 208 & 2.77 & 0.0506 & 0.9249 & \\
\hline
\end{tabular}

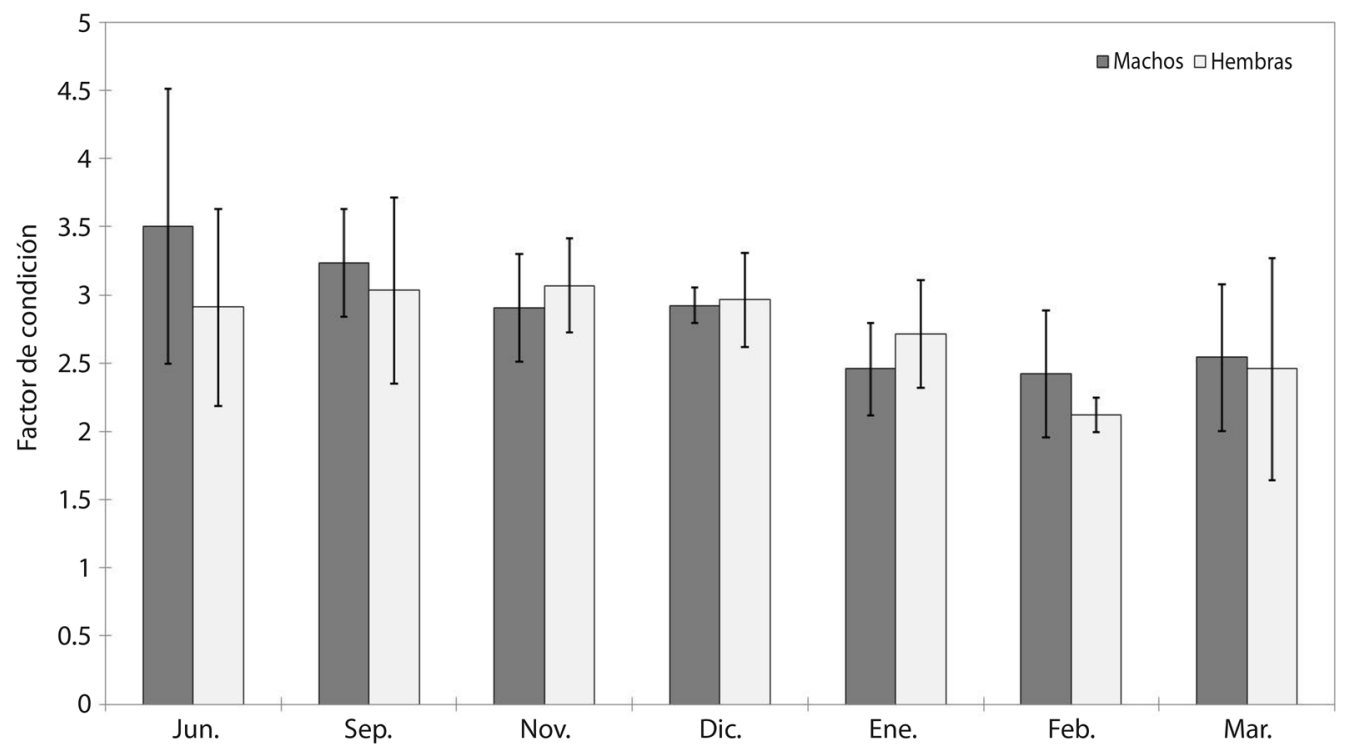

Fig. 7. Comportamiento del Factor de Condición (+ / - d.s.) para machos y hembras de Opsanus beta en el SLA por mes. Fig. 7. Behavior of the Condition Factor (+/ - s.d.) for males and females of Opsanus beta in the SLA for month.

Franco-López, Abarca-Arenas, Silva-López, Valero-Pacheco, \& Zamudio-Arciniega, 2012).

Se registró un número alto de organismos machos con relación al de las hembras, Keiti (2013) presenta resultados similares sobre esta misma especie en Brasil. Las mayores capturas de machos en los meses de abril, diciembre y marzo, podrían estar relacionados con su temporada y comportamiento reproductivo en el
SLA y su probabilidad de ser capturados por el papel que juegan al proteger el área de desove, como lo mencionan Swartz y Van Engel (1968). La proporción de sexos general favoreció a los machos en los meses de abril, junio, diciembre, febrero, marzo y en el total de los organismos capturados, se presentan diferencias significativas de la relación 1:1 esperada, por su parte los meses de noviembre y enero que favorecieron 

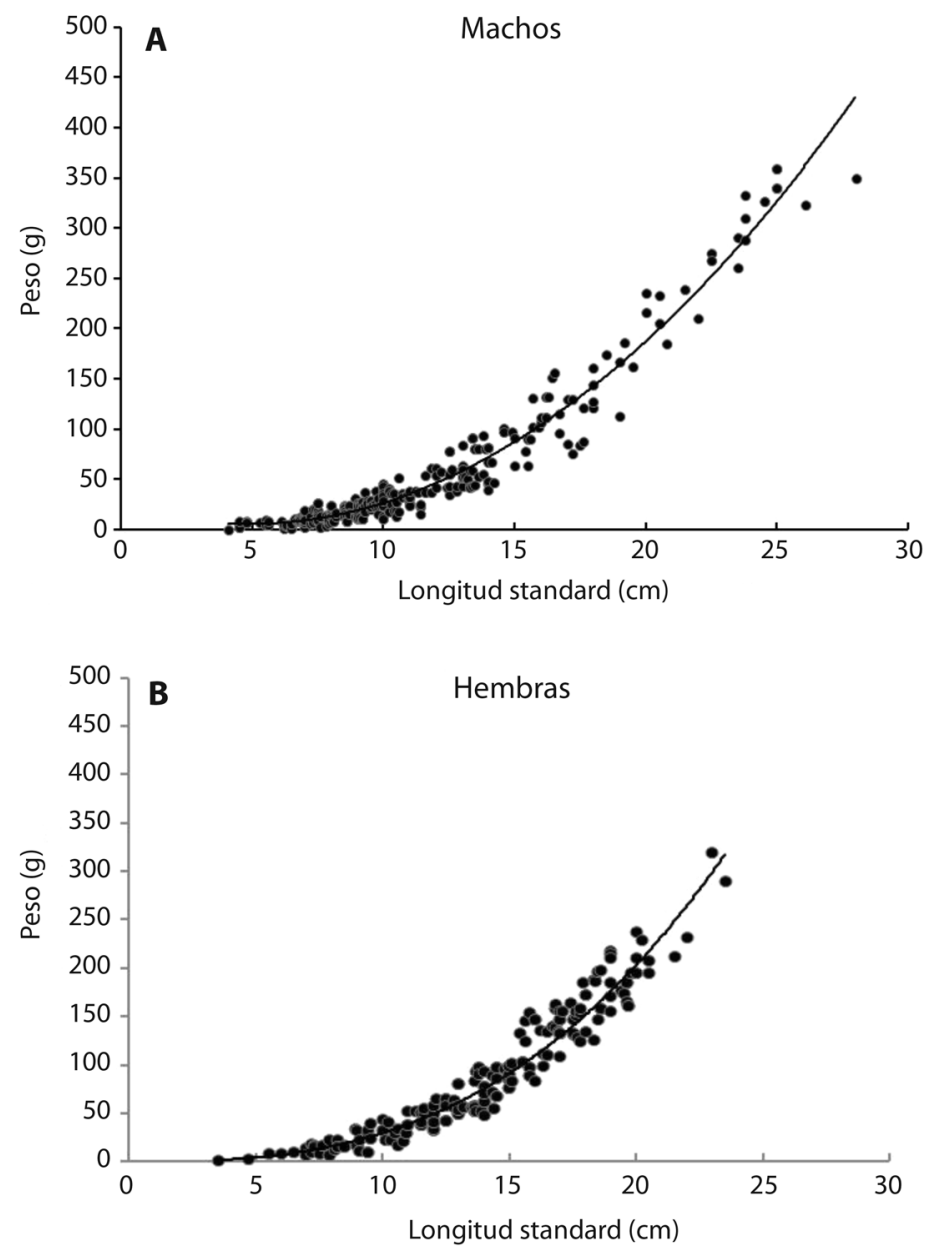

Fig. 8. Relación Peso-Longitud para machos (A) y hembras (B) de Opsanus beta en el SLA período 2008-2012.

Fig. 8. Weight-Length Relationship for males (A) and females (B) of Opsanus beta in the SLA period 2008-2012.

a las hembras no se presentaron diferencias significativas de la relación $1: 1$. Al respecto, Swartz y Van Engel (1968) y Schwartz y Dutcher (1963), mencionan que la proporción de sexos también fue favorable para los machos de la especie Opsanus tau, así como lo que reportan Palazón, Arias y Sarasquete (2001), quienes trabajaron con Halobatrachus didactylus otra especie de la familia (Batrachoididae), y registraron un comportamiento similar que favoreció a los machos en la proporción de sexos. Una posible explicación de este comportamiento de una mayor captura de machos en relación con las hembras, la presentan Swartz y Van Engel (1968), quienes mencionan que esto puede ser debido a que los machos son los encargados de cuidar el sitio de desove, y por ello son más vulnerables a ser capturados.

Durante los meses de noviembre a febrero, los machos registraron las longitudes y pesos más altos, en donde alcanzan los $28 \mathrm{~cm}$ y un peso de 426.8 gramos contra los $23.5 \mathrm{~cm}$ y los 320.7 gramos que se presentó en las hembras en los mismos meses, al respecto, McDermott (1964) y Walsh, Bedolla y Mommsen (1989), también reportan que los machos de esta especie presentaron un peso mayor que las hembras. Un comportamiento similar donde las tallas de los machos son mayores en relación con las hembras, lo reportan Newman, Gruber y Handy 
(2004), para Opsanus phobetron, y señalan que este comportamiento puede explicarse por la maduración sexual temprana en las hembras.

En relación con las preferencias alimenticias de la especie, los resultados muestran que su dieta está compuesta de 13 tipos alimenticios, entre los que destacan los crustáceos (Callinectes sp., Cardisoma sp., Macrobrachium sp), gasterópodos y bivalvos (Neritina virginea, Rangia sp., Mytilopsis sp.) y peces, (Citharithys spilopterus, Gobionellus oceanicus, peces no identificados y Opsanus beta), estos son consumidos de igual manera por juveniles como por adultos. Un aspecto a destacar es el comportamiento caníbal en esta especie, que hasta la fecha no había sido reportada en la literatura, pero que con base en los análisis de contenido estomacal, permitió reconocer que los organismos adultos llegan a consumir juveniles de la misma especie en la temporada de secas, particularmente en los meses de marzo y abril. Al respecto, Cogliati et al. (2015) reportan que Porichthys notatus representante de la familia Batrachoididae presenta un comportamiento caníbal sobre embriones de su misma especie, aspecto que relacionan con el cuidado parental del nido, pero que difiere del comportamiento de O. beta en el SLA que consume juveniles y que puede concordar con lo reportado por Palmer, Deffenbaugh y Mensinger (2005), quienes mencionan que el carácter depredador de Opsanus tau, especie emparentada con Opsanus beta, se ve favorecido por la rápida respuesta en movilidad que muestra en los lugares que habita, atacando a diversas especies que comparten el hábitat con esta especie. Por otro lado, nuestros resultados contrastan con lo que reportan Canto-Maza y Vega-Cendejas (2007), quienes mencionaron que para la especie Opsanus phoebetron en la laguna costera de Chelem, en Yucatán, los juveniles tienen una dieta diferente a la de los adultos. La composición en los items alimenticios registrados para esta especie, coincide con los reportes de autores que han trabajado con una especie emparentada como es Opsanus tau en la costa este de Estados Unidos (Wilson, Dean, \& Radtke, 1982; Bisker, Gibbons, \& Castagna,
1989; Price \& Mensinger, 1999; Grabowski, 2004) y que mencionan que los tipos alimenticios que consume esta especie son Callinectes sapidus, miembros de la familia Xanthidae, y bivalvos principalmente, o lo reportado por Collete (1974) para Sanopus splendidus, para quien reporta una dieta compuesta por crustáceos, moluscos y peces.

Algunos autores señalan que hay una relación entre la temporada de reproducción, el desove y la latitud (Cushing, 1975; Blaxter \& Hunter, 1982), indican que en latitudes altas, al presentarse un periodo cálido muy breve, las especies desarrollan un periodo de desove corto, en tanto que en zonas más cercanas al Ecuador, los periodos de reproducción son más prolongados o pueden presentar desoves parciales que pueden extenderse a lo largo del año (Cushing, 1975). El comportamiento reproductivo registrado para esta especie, al tomar como base las etapas de maduración gonádica propuestas por Nikolsky (1963), indican que el proceso de maduración para machos y hembras de Opsanus beta es más evidente en el mes de noviembre, es decir a inicio de la temporada de Nortes y alcanzando su máximo en febrero y marzo para el caso de los machos, y solo alcanza el mes de febrero para las hembras. El comportamiento registrado coincide con estudios previos elaborados en Florida y mencionados por Cathleen (2005), que mencionan que esta especie desova en los meses de febrero y marzo, así como los estudios reportados por Keiti (2013) en Brasil, y el de Gallardo, Martinez y Lezina (2004) en Tecolutla, donde encuentran que las etapas de maduración más avanzadas para las hembras de Opsanus beta se dan entre octubre y febrero, y de octubre a abril, respectivamente.

Relacionado con el proceso de maduración gonádico, se han desarrollado diversos índices que ayudan a explicar la dinámica en la utilización de la energía consumida a lo largo del año por el pez en órganos como gónadas, hígado y masa corporal, un ejemplo es el IGS, el IHS y el FC (Collins \& Anderson, 1995). Los resultados obtenidos del IGS en machos y hembras de $O$. beta, confirman que el periodo 
reproductivo de $O$. beta abarca los meses de noviembre a abril en el sistema lagunar de Alvarado, y que después del periodo reproductivo, se incrementa el número de organismos, inmaduros y en proceso de maduración, así como de desovados. Se ha señalado que el IGS se relaciona de forma inversa con el FC y al IHS, como resultado de la demanda de energía por parte del pez para procesos reproductivos. El comportamiento del IHS así como el factor de condición FC para Opsanus beta, en relación con el IGS, coincide con el comportamiento descrito, al presentar un comportamiento inverso, lo cual puede indicar una variación en el peso del hígado como resultado de proceso de almacenamiento y transferencia de proteínas y lípidos asociados con el esfuerzo reproductivo (Santos, Hawkinis, \& Nash, 1996). Por su parte, en relación con el Factor de Condición (FC), las variaciones en el peso corporal indicarían que la energía almacenada en forma de lípidos y proteínas es utilizada para hacer frente a los requerimientos de energía en los períodos donde se reduce la alimentación (Jobling, 1995; Maddock \& Burton, 1999). Como resultado de esto, a medida que se incrementa el tamaño de la gónada, se incrementa el índice Gonadosomático (IGS), se reduce el consumo de alimento y se provoca un ayuno durante la maduración gonadal que lleva a la reabsorción de proteínas musculares que son utilizadas para la síntesis de vitelo (Bulow, Zeman, Winningham, \& Hudson, 1981).

El número de ovocitos por hembra presentó variaciones no relacionadas con la talla, por ejemplo en hembras en etapa de maduración IV, con una longitud estándar $<14 \mathrm{~cm}$, el número de ovocitos fue mayor que en hembras con tallas $>18 \mathrm{~cm}$, mientras que en la etapa de maduración más avanzada, el número de ovocitos es mayor a medida que la longitud estándar aumenta. De esta forma se puede decir que $O$. beta presenta una fecundidad en el SLA de 96 a 428 ovocitos por hembra. Se reconoce que la fecundidad por gónada para $O$. beta es relativamente baja y es una característica de la familia Batrachoididae (Breder 1941; Collette \& Ruso, 1981). Sin embargo, esta baja fecundidad se compensa por el cuidado parental hacia las crías, lo que aumenta la probabilidad de sobrevivencia de la descendencia. Además, los huevos son bentónicos y adheridos a objetos sumergidos, lo que facilita el cuidado parental, esta estrategia reproductiva es típica de los miembros de la familia (Breder \& Rosen, 1966). Gallardo et al., (2004) reportan para esta especie en el estuario de Tecolutla un fecundidad de 79 a 518 ovocitos por hembra. Por su parte, Palazón et al. (2001), reportan para Halobatrachus didactylus (Batrachoididae), un rango de fecundidad de 227 a 1233 huevos por hembra y mencionan que este número está relacionado con el largo y peso del cuerpo del pez.

Wilbur y Robinson (1960), reportan para $O$. tau en Maryland un intervalo de tallas que varía de 16.6 a $32.3 \mathrm{~cm}$, intervalo muy similar al que presenta $O$. beta en este sistema para ambos sexos. Los resultados de la relación peso-longitud para cada sexo y temporada, permitió reconocer para esta especie un mayor coeficiente de alometría (b) para los machos que para las hembras. Sin embargo, en ambos casos, se obtuvo que Opsanus beta en el SLA presenta un crecimiento de tipo alométrico negativo, al respecto Dovel (1960), menciona que la diferencia en el tipo de crecimiento puede deberse al patrón de desarrollo larvario y la forma que adquiere el pez en su etapa adulta, relacionado con el hábitat que ocupa.

Opsanus beta es una especie residente en el sistema lagunar de Alvarado a la que se le reconoce utilizar diferentes componentes del hábitat y donde las variaciones en las condiciones fisicoquímicas del sistema, no afectan su distribución espacial y ocupación de espacios, lo que ha permitido adaptarse a las condiciones variantes de la temperatura del agua, salinidad y oxígeno disuelto, que se presentan en las diferentes temporadas climáticas del sistema, y para poder completar su ciclo de vida.

El análisis de los parámetros biológicos permitió reconocer que los machos desarrollan tallas mayores que las hembras y que la proporción de sexos también favoreció a los machos, aspectos que coinciden con los reportes de otros autores en otras localidades del golfo 
de México. El uso que hace de las especies que viven en este sistema lagunar como alimento, hace que su dieta se componga de 13 tipos alimenticios donde destacan los crustáceos, moluscos y peces como los tipos principales. Asimismo, se reconoció que esta especie se comporta como caníbal de individuos jóvenes.

El comportamiento del IGS, IHS, y FC indicaron que esta especie alcanza sus máximas etapas de maduración entre los meses de noviembre a marzo y que su fecundidad varía de 96 a 428 ovocitos por hembra, lo que coincide con lo que reportan diversos autores para otras especies de la familia Batrachoididae. Adicionalmente, se reconoció que el tipo de crecimiento que desarrolla esta especie en el SLA es alométrico negativo.

Es importante generar información biológica y ecológica de las especies que permita conocer a detalle las formas en que interactúan y participan en los diferentes procesos que se desarrollan en los ambientes estuarinos, que pueda además ser útil para afinar las propuestas de manejo y aprovechamiento sustentable de los ecosistemas costeros, en especial los más vulnerables.

\section{AGRADECIMIENTOS}

Los autores agradecen a la División de Investigación de la FES-Iztacala de la UNAM, al Convenio de intercambio académico de la UNAM con la Universidad Veracruzana, así como al proyecto Recursos Acuáticos por el apoyo brindado para el desarrollo de este trabajo. Adicionalmente, a Tomas Corro Ferreiro por su valiosa ayuda en la colecta de los organismos, así como a los revisores anónimos por sus observaciones que contribuyeron a mejorar el trabajo.

\section{RESUMEN}

Opsanus beta es un habitante permanente del Sistema Lagunar de Alvarado, a pesar de participar en la transferencia energética entre los distintos componentes, no hay información sobre los aspectos ecológicos y reproductivos de esta especie. El presente trabajo se desarrolló, con la finalidad de evaluar aspectos de la biología como estacionalidad, alimentación, proporción de sexos, índice gonadosomático, hepatosomático y factor de condición para ambos sexos, así como la fecundidad y la relación peso-longitud, durante el período de abril 2008 a diciembre 2012. Se registraron los parámetros de temperatura ambiental y del agua, profundidad, salinidad, oxígeno disuelto y $\mathrm{pH}$ en cada uno de los seis sitios de recolecta que se ubicaron en el sistema. El análisis de los parámetros ambientales no mostró diferencias significativas entre los años del período de estudio, la salinidad registrada a lo largo del estudio correspondió a un ambiente oligohalino, el resto de los parámetros fisicoquímicos presentó variaciones que pueden explicarse por el patrón climático en el área. Se capturó un total de 519 organismos, 311 (59.9\%) machos y 208 (41.1\%) hembras, el comportamiento de la longitud patrón en los distintos meses muestra que en los machos, la longitud promedio es mayor que en las hembras. El espectro trófico se compone de 13 tipos alimenticios, destacan los crustáceos, moluscos y peces, adicionalmente se observó la práctica de canibalismo en los meses de marzo y abril. Los estadios de maduración gonádica más avanzados se registraron en los meses de noviembre a marzo para los machos, y de noviembre a febrero para las hembras. El IGS registró los valores más altos en los meses de noviembre a abril, el IHS y el FC presentaron un comportamiento inverso al IGS. La fecundidad de esta especie varía de 96 a 428 ovocitos por hembra. La relación peso-longitud por sexo y temporada presentó una mayor tasa de crecimiento (b) para los machos que para las hembras, y un crecimiento de tipo alométrico negativo. La información biológica y ecológica de $O$. beta en el SLA permite reconocer que esta especie es importante por el papel ecológico que juega en la estructura y dinámica de sus comunidades acuáticas y puede ser utilizada en la propuesta de manejo y aprovechamiento sustentable de este ecosistema.

Palabras clave: Opsanus beta, índices reproductivos, alimentación, crecimiento.

\section{REFERENCIAS}

Arceo, C. D., Franco, L. J., Gretchen, L. W., \& Chávez, L. R. (2004). Trophic comparison of two species of needlefish (Belonidae) in the Alvarado Lagoonal System, Veracruz, México. Gulf and Caribbean Research, 16, 81-88.

Bagenal, T. B. (Ed). (1978). Methods for assessment of fish production in freshwater. Oxford, UK: Blackwell Scientific Publication.

Balon, E. K. (1975). Reproductive guilds of fishes: A proposal and definition. Journal of the Fisheries Research Board of Canada, 32, 821-864.

Bisker, R., Gibbons, M., \& Castagna, M. (1989). Predation by the oyster toadfish Opsanus tau (Linnaeus) 
on blue crabs and mud crabs, predators of the hard clam Mercenaria mercenaria (Linnaeus 1758). Journal of Shellfish Research, 8(1), 25-31.

Blaxter, J. H. S., \& Hunter, J. R. (1982). The biology of clupeoid fishes. Advances in Marine Biology, 20, 3-194.

Breder, C. (1941). On the reproduction of Opsanus beta. Goode \& Bean. Zoologica,l 26, 229-232.

Breder, C. M., \& Rosen, D. E. (1966). Modes of reproduction in fishes. Neptune City, New Jersey: T. F. H. Publications.

Bulow, F. J., Zeman, M. E., Winningham, J. R., \& Hudson, W. F. (1981). Seasonal variations in RNA-DNA ratios and in indicators of feeding, reproduction, energy storage, and condition in a population of bluegill, Lepomis macrochirus Rafinesque. Journal of Fish Biology, 18, 237-244.

Caires, R. A., Pichler, H. A., Spach, H. L., \& Ignacio, J. M. (2007). Opsanus brasiliensis (Rotundo, Spinelli \& Zavalla-Camin, 2005) (Teleostei: Batrachoidiformes: Batrachoididae), a junior synonym of Opsanus beta (Goode \& Bean, 1880), with notes on its occurrence in the Brazilian coast. Biota Neotropica, 7(2), 135140. Retrieved from http://www.biotaneotropica.org. br/v7n2/pt/abstract?article+bn02307022007

Canto-Maza, W. G., \& Vega-Cendejas, M. E. (2007). Distribución, abundancia y preferencias alimenticias del pez sapo Opsanus phobetron (Batrachoididae) en la laguna costera de Chelem Yucatán, México. Revista de Biología Tropical, 55(3-4), 979-988.

Cathleen, B. (2005). Opsanus beta/Species Account. Florida, USA: Florida Museum of Natural History. Retrieved from https://www.flmnh.ufl.edu/fish/discover/ species-profiles/opsanus-beta

Chandler, C. R., Sanders, R. M. Jr., \& Landry, A. M. Jr. (1985). Effects of three substrate variables on two artificial reef fish communities. Bulletin of Marine Science, 37, 129-142.

Cogliati, K. M., Danukarjanto, C., Pereira, A. C., Lau, M. J., Hassan, A., Mistakidis, A. F., ... Balshine, S. (2015), Diet and cannibalism in plainfin midshipman Porichthys notatus. Journal of Fish Biology, $86,1396-1415$

Collette, B., Aiken, K. A., \& Polanco-Fernández, A. (2015). Opsanus beta. United Kingdom: The IUCN Red List of Threatened Species 2015. Retrieved from http://dx.doi.org/10.2305/IUCN.UK.2015-4.RLTS T190257A1946238.en

Collette, B. B. (1974). A review of the coral Toadfishes of the genus Sanopus with descriptions of two new species from Cozumel island, Mexico. Proceedings of the Biological Society of Washington, 87(18), 185-204. Retrieved from https://archive.org/ stream/proceedingsofbi871974biol/proceedingsofbi871974biol_djvu.txt

Collette, B. B., \& Russo, J. L. (1981). A revision of the scaly toadfishes, genus Batrachoides, with descriptions of two new species from the Eastern Pacific. Bulletin of Marine Science, 31, 197-233.

Collins, A. L., \& Anderson, T. A. (1995). The regulation of endogenous energy stores during starvation and refeeding in the somatic tissues of the golden perch. Journal of Fish Biology, 47, 1004-1015.

Contreras, E. F., \& Warner, G. B. (2004). Ecosystem characteristics and management considerations for coastal wetlands in Mexico. Hydrobiologia, 511, 233-245.

Costanza, R., d'Arge, R., de Groot, R., Farber, S., Grasso, M., Hannon, B., ... van den Belt, M. (1997): The value of the world's ecosystem services and natural capital. Nature, 387(6630), 253-260.

Cushing, D. H. (1975). Marine ecology and fisheries. Oxford, Great Britain: Cambridge University Press.

Dovel, W. (1960). Larval development of the oyster toadfish, Opsanus tau. Chesapeake Science, 1, 187-195.

Le Cren, E. D. (1951). The length-weight relation and seasonal cycle in gonad weight and condition in the perch, Perca fluviatilis. Journal of Animal Ecology, 20(2), 201-219.

Franco-López, J., Abarca-Arenas, L. G., Silva-López, G., Valero-Pacheco, E., \& Zamudio-Arciniega, R. (2012). Seasonal Analysis of the Ichthyofauna at tree subsystems of the Alvarado lagoon, Veracruz, Mexico. In D. Thangadurai, C. A. Busso, L. G. Abarca, \& S. Jayabalan, (Eds.), Frontiers in Biodiversity Studies (pp. 213-240). New Delhi. India: I.K. International Publishing House, Pvt. Ltd.

Gallardo, T. A., Martinez, P. J., \& Lezina, B. J. (2004). Reproductive structures and early life history of the gulf toadfish, Opsanus beta, in the Tecolutla Estuary, Veracruz, Mexico. Gulf and Caribbean Research, 16, 109-113.

Grabowski, J. H. (2004). Habitat complexity disrupts predator-prey interactions but not the trophic cascade on oyster reefs. Ecology, 85, 995-1004.

Jobling, M. (1995). Environmental biology of fishes. London, England: Chapman \& Hall.

Keiti, N. J. (2013). Biologia reprodutiva e alimentar da espècie exòtica Opsanus beta (Teleostei: Batrachoididae) no complexo estuarino de Paranagùa, Paranà, Brasil (Tese de Mestre em Ecologia e Conservação). Universidade Federal do Paraná, Brasil.

Maddock, D. M., \& Burton, M. P. M. (1999). Gross and histological observations of ovarían development 
and related condition changes in American plaice. Journal of Fish Biology, 53, 928-944.

Malca, E., Barimo, J. F., Serafy, J. E., \& Walsh, P. J. (2009). Age and growth of the gulf toadfish Opsanus beta based on otolith increment analysis. Journal of Fish Biology, 75, 1750-1761.

McDermott, J. J. (1964). Food habits of the toadfish, Opsanus tau (L.) in New Jersey waters. Proceedings of the Pennsylvania Academy of Science, 38, 64-71.

Morán, S. A., Martínez, F. L. A., Chávez, L. R., Franco, L. J., Bedia, S. C. M., Contreras, E. F., ... \& Peterson, M. S. (2005) Seasonal and spatial patterns in salinity, nutrients, and chlorophyll a in the Alvarado Lagoonal System, Veracruz, México. Gulf and Caribbean Research, 17(1), 133-143.

Newman, S. P., Gruber, S. H., \& Handy, R. D. (2004), The scarecrow toadfish: habitat, abundance and size at maturity at Bimini, Bahamas. Journal of Fish Biology, 64, 248-252.

Nikolsky, G. V. (1963). The ecology of fishes. New York, USA: Academy Press.

Palazón, F. J. L., Arias, A. M., \& Sarasquete, C. (2001). Aspects of the reproductive biology of the toadfish, Halobatrachus didactylus (Schneider, 1801) (Pisces: Batrachoididae). Scientia Marina, 65(2), 131-138.

Palmer, M. L., Deffenbaugh, M., \& Mensinger, A. F. (2005). Sensitivity of the anterior lateral line to natural stimuli in the oyster toadfish, Opsanus tau (Linnaeus). The Journal of Experimental Biology, 208, 3441-3450.

Pereira, T. J., Silva, G., Costa, M. J., \& Costa, J. L. (2011). Life strategies of Halobatrachus didactylus (Bloch and Schneider, 1801) in the Tagus estuary: comparison among different morphotypes. Estuarine, Coastal and Shelf Science, 93, 328-335

Price, N. N., \& Mensinger, A. F. (1999). Predator-prey interactions of juvenile toadfish, Opsanus tau. The Biological Bulletin, 197, 246-247.

Ramos, H. M. (1997). Alvarado, Apuntes Históricos y Geográficos. México: Gobierno del Estado de Veracruz de la Llave.

Rodríguez, G. M. (1992). Técnicas de Evaluación Cuantitativa de la Madurez Gonádica en Peces. México: AGT Editores.

Roux, C. (1986). Batrachoididae. In P. J. P. Whitehead., M. L. Bauchot, J. C. Hureau \& E. Tortonese (Eds.),
Fishes of the North-eastern Atlantic and the Mediterranean (pp. 1360-1361). Vol. III. Paris: UNESCO.

Santos, R. S., Hawkinis, S. J., \& Nash, R. D. (1996). Reproductive phenology of the Azorean rock pool blenny a fish alternative mating tactics. Journal of Fish Biology, 48, 842-858.

Schwartz, F. J., \& Dutcher, B. W. (1963). Age, growth, and food of the Oyster Toadfish near Solomons, Maryland. Transactions of the American Fisheries Society, 92, 170-173.

Smith, C. L. (1997). National Audubon Society field guide to tropical marine fishes of the Caribbean, the Gulf of Mexico, Florida, the Bahamas, and Bermuda. New York, USA: Alfred A. Knopf.

Swartz, R. C., \& Van Engel, W. A. (1968). Length, Weight, and Girth Relations in the Toadfish, Opsanus tau. Chesapeake Science, 9, 249-253.

Tupper, M., \& Boutilier, R. G. (1995). Effects of habitat on settlement, growth, and postsettlement survival of Atlantic cod (Gadus morhua). Canadian Journal of Fisheries and Aquatic Sciences, 52, 1834-1841.

Walsh, P. J., Bedolla, C., \& Mommsen, T. P. (1989). Scaling and sex-related differences in toadfish (Opsanus beta) sonic muscle enzyme activities. Bulletin of Marine Science, 45, 68-75.

Wilbur, C. G., \& Robinson, P. F. (1960). The correlation of length, weight, and girth in the toadfish Opsanus tau. Chesapeake Science, 1(2), 122-123.

Wilson, C. A., Dean, J. M., \& Radtke, R. (1982). Age, growth rate and feeding habits of the oyster toadfish, Opsanus tau (Linnaeus) in South Carolina. Journal of Experimental Marine Biology and Ecology, 62, 251-259.

Wood, E. J. F., Odum, W. E., \& Zieman, J. C. (1969). Influence of sea grasses on the productivity of coastal lagoons. En A. A. Castañares \& F. B. Phleger (Eds.), (1969) Coastal lagoons (pp. 495-502). México, D. F: Universidad Nacional Autónoma de México, Ciudad universitaria.

Yáñez-Arancibia, A. (1978). Patrones ecológicos y variación cíclica de la estructura trófica de las comunidades nectónicas en lagunas costeras del Pacífico de México. Anales del Centro de Ciencias del Mar y Limnología-UNAM, 5(1), 287-306.

Zar, J. H. (2010). Biostatistical analysis. New Jersey, USA: Prentice Hall. 